\title{
Quaderni
}

QUADERNI Communication, technologies, pouvoir

\section{La recherche indépendante aux États-Unis et en France : le Council on Foreign Relations et l'Ifri}

\section{François Vergniolle de Chantal}

\section{(2) OpenEdition}

\section{Édition électronique}

URL : http://journals.openedition.org/quaderni/507

DOI : 10.4000/quaderni.507

ISSN : 2105-2956

Éditeur

Les éditions de la Maison des sciences de l'Homme

\section{Édition imprimée}

Date de publication : 5 octobre 2009

Pagination : 49-56

\section{Référence électronique}

François Vergniolle de Chantal, « La recherche indépendante aux États-Unis et en France : le Council on Foreign Relations et l'Ifri », Quaderni [En ligne], 70 | Automne 2009, mis en ligne le 05 octobre 2011, consulté le 10 décembre 2020. URL : http://journals.openedition.org/quaderni/507 ; DOI : https:// doi.org/10.4000/quaderni.507 


\section{$D$ ossier}

la recherche indépendante aux États-Unis et en France : le Council on Foreign Relations et l'Ifri

\section{François Vergniolle de Chantal}

Maître de Conférences Université de Bourgogne
Les différentes contributions de ce dossier ont toutes souligné la spécificité des États-Unis et des centres de recherche indépendants (think tanks). Ces derniers s'inscrivent dans un cadre social et politique classiquement analysé par Alexis de Tocqueville en son temps, celui d'une vitalité associative résultant de la faiblesse de l'Etat fédéral : "Les associations politiques qui existent aux États-Unis ne forment qu'un détail au milieu de l'immense tableau que l'ensemble des associations y présente. (...) Les Américains s'associent pour donner des fêtes, fonder des séminaires, bâtir des auberges, élever des églises, répandre des livres (...). S'agit-il enfin de mettre en lumière une vérité ou de développer un sentiment (...), ils s'associent $»^{1}$.

Dans le domaine de la définition de la politique étrangère, la configuration américaine est encore plus surprenante. Au moment de leur fondation, les États-Unis, par réaction au modèle européen où le Monarque décide seul de la guerre et de la diplomatie, se dotent d'un système plus ouvert où le Congrès et la Présidence se partagent les décisions. Ainsi, grâce au Congrès, l'opinion publique est une contrainte très importante dans la définition de la politique étrangère du pays. Les think tanks possèdent donc une légitimité incontestable en tant qu'acteurs de la société civile participant à la formulation de la diplomatie nationale. Il s'agit ici d'illustrer cette spécificité sur la base d'une description du Council on Foreign Relations (CFR), une des institutions les plus prestigieuses de la scène américaine, pour ensuite en venir à la situation en France au travers du cas de l'Ifri (Institut Français des Relations Internationales) dont l'ambition affichée est d'être un think tank européen. 
Le Council on Foreign Relations est sans doute le plus connu des centres de réflexion en politique internationale ; il est de plus, et comme le dit Justin Vaisse, « inclassable » de par « l'osmose » qui $\mathrm{y}$ règne entre universitaires, hauts fonctionnaires, diplomates, et leaders politiques ${ }^{2}$. Le Council est donc au cœur de l'establishment de politique étrangère aux États-Unis. Il est l'un des plus anciens think tanks du pays. Il fut en effet fondé en 1921, dans le sillage des bouleversements mondiaux de la guerre, par des intellectuels et des « praticiens » fortement imprégnés des idées « progressistes » du début de siècle, notamment Elihu Root ${ }^{3}$. L'origine historique du CFR est donc à trouver dans l'intensité diplomatique de l'après-guerre mondiale. Entre 1917 et 1918, un groupe d'environ 150 universitaires fut chargé par Woodrow Wilson - lui-même universitaire et encore seul président américain à posséder une thèse $(P h D)$ - de réfléchir aux options pour organiser la paix. Ce groupe - au sein duquel se trouvaient des conseillers présidentiels, comme Edward M. House, ou des intellectuels reconnus, comme Walter Lipmann - se rencontrait à New York, et produisit au final plus de 2000 documents analysant le contexte nouveau et ses conséquences. L'ensemble de cette production eut une influence forte sur les 14 points que Wilson présenta aux Européens en janvier 1919. Lors des négociations, les conseillers américains et leurs collègues britanniques décidèrent de pérenniser leur collaboration en les institutionnalisant : l'idée du Conseil était née, avec comme pendant Chatham House à Londres. Les deux institutions se développèrent cependant de façon indépendante, la version britannique devenant le Royal Institute for International Affairs, tout en conservant des liens informels de l'autre côté de
l'Atlantique. Côté américain, les experts, à leur retour d'Europe, furent conviés à participer à des rencontres avec des hommes d'affaires newyorkais par l'entremise d'Elihu Root. À la suite de ces rencontres, le CFR fut finalement créé en 1921 par 108 personnes. L'année suivante, le Council se dota de la revue Foreign Affairs. Le financement, initialement assuré par 26 entreprises différentes, a été marqué par les dons de John Rockefeller Jr., fils du célèbre industriel.

L'objectif initial était de développer la compréhension que l'élite avait des questions internationales. Le choc du rejet de la SDN fut bien évidemment le catalyseur qui précipita la volonté d'institutionnaliser le centre et de lutter contre le sentiment isolationniste. Cet agenda internationaliste fut la grande caractéristique du CFR pendant l'entre-deux-guerres dans un contexte où l'Amérique se repliait largement sur elle-même malgré son nouveau rôle d'acteur incontournable sur la scène internationale. De ce point de vue, il convient sans doute de relativiser l'idée selon laquelle le CFR serait un acteur décisif dans la formulation de la politique étrangère du pays ${ }^{4}$. En revanche, avec la montée des menaces en Europe, le CFR devient une source d'analyses pour les autorités. Ainsi en 1939 et dans les cinq années qui suivent, le CFR publia les War And Peace Studies, financées entièrement par la Rockefeller Foundation, mais secrètes à tel point que tous les membres du CFR n'étaient pas au courant de la rédaction de ces études. Si on a là une illustration de l'osmose entre les milieux officiels et le CFR, la consécration du Council et de Foreign Affairs vint après la Seconde Guerre mondiale. C'est en juillet 1947 que George Kennan publia en effet une version modifiée de son fameux télégramme 
dans Foreign Affairs («The Sources of Soviet Conduct ») en utilisant le terme « endiguement» («containment»). Des initiatives aussi fondamentales que le Plan Marshall ou l'OTAN furent aussi partiellement anticipées au sein du CFR. Dès lors, le CFR profita à plein de son statut quasi officiel. Il avait un monopole presque total sur la pensée en matière de politique étrangère, notamment sur des thèmes comme la dissuasion, le contrôle des armements, la prolifération nucléaire, mais aussi pour la guerre du Vietnam. À la fin de la décennie, le CFR et Foreign Affairs furent cependant en décalage par rapport à l'opinion. Leur succès des vingt dernières années avait eu un prix : le CFR avait perdu en effet la vitalité qui était la sienne avant la Seconde Guerre mondiale. C'est un consensus paresseux qui s'installa dans les années soixante, précipitant la naissance d'alternatives - à commencer par Foreign Policy en 1970, mais aussi le développement de concurrents comme la RAND Corporation (1948), le « Policy Planning Staff» (1947) au sein de l'Exécutif, ou encore le CSIS (Center for Strategic and International Studies créé en 1962) et sa revue le Washington Quarterly. Depuis les années 90, la revue Foreign Affairs a pris un nouveau départ, en limitant par exemple le nombre de textes « officiels », tout en ouvrant les colonnes à des visions nouvelles, parfois provocantes : c'est ainsi qu'en 1993 Samuel Huntington publia son « Clash of Civilizations? » dans Foreign Affairs ou encore que Condoleezza Rice écrivit «Promoting the National Interest » en 2000.

Comme tous les think tanks, le Council on Foreign Relations se déclare «non partisan », c'est-à-dire indépendant des partis politiques, et n'est pas non plus une institution à but lucratif. Au contraire, le CFR est enregistré sous un statut fiscal dit 501(c)(3), c'est-à-dire une association à but non-lucratif («not-forprofit organization $»)^{5}$. Depuis 1917, les dons à ces institutions ouvrent droit à déduction fiscale. Ce statut fiscal est important en ce qu'il permet de faire la différence avec les groupes de pression (lobbies). Les think tanks bénéficient en effet d'exonérations fiscales - notamment pour l'impôt local sur les sociétés et l'impôt fédéral sur le revenu - ce qui n'est pas le cas des lobbies. Le CFR a son siège à New York, mais possède une délégation à Washington DC et d'autres institutions affiliées dans le pays. Il revendique 4300 membres; comme l'indique le site du Council, il y a différentes formules pour être membres du CFR : à vie, ou bien pour 5 ans. L'adhésion est réservée aux citoyens américains - ou bien aux résidents permanents candidats à la naturalisation - entre 30 et 36 ans. L'adhésion à vie doit être parrainée par écrit par un membre du CFR et soutenue par au moins trois autres. Il y a également des adhésions d'entreprises - 250 actuellement - réparties en trois catégories : " basic »- " premium » (plus de 25000 dollars) - et « President's Circle » (plus de 50000 dollars). Cela ouvre le droit à écouter les responsables intervenant au sein du CFR, soit pour les séances officielles, soit pour des rencontres privées, fermées au public. L'activité intellectuelle du Conseil est assurée par les 91 chercheurs qui y travaillent à plein temps.

Le CFR publie Foreign Affairs tous les 15 jours. Le CFR vise à promouvoir les débats sur la politique étrangère des États-Unis en associant professionnels, hauts fonctionnaires, journalistes, et universitaires. Il cherche donc 
à être un lieu de rencontres pour irriguer la diplomatie américaine avec de nouvelles idées, tout en informant l'opinion publique. Il possède en son sein un programme distribuant des bourses de recherche pour mener des projets sur les thèmes intéressant le CFR (le « David Rockefeller Program ») et qui portent sur les défis auxquels les États-Unis doivent faire face aussi bien que sur les problèmes fondamentaux du monde contemporain. Des "task force» sont organisées sur des thèmes du moment et gèrent conférences, auditions et publications. Le Conseil doit son audience à l'intensité des liens avec les milieux officiels - plus d'une douzaine de secrétaires d'État sont passés par le CFR et sept présidents y ont fait des discours - mais aussi les médias, voire les services secrets.

Depuis 2003, le CFR est présidé par Richard N. Haas, ancien directeur du « Policy Planning Staff » et ancien collaborateur du président G.H.W. Bush pour le Moyen-Orient (19891993). On trouve également, dans le conseil d'administration, les noms de Robert E. Rubin, ancien secrétaire du Trésor sous Clinton (19951999), Madeleine K. Albright ambassadeur des États-Unis à l'ONU (1993-1997) puis première femme Secrétaire d'Etat (1997-2001), Joseph S. Nye Jr. (universitaire bien connu pour ses travaux sur le «soft power »), Colin L. Powell (Secrétaire d'État de 2001 à 2005), et Christine Todd Whitman (gouverneur du New Jersey de 1994 à 2001, puis directrice de l'« Environmental Protection Agency » de 2001 à 2003).

En Europe, la configuration est fort différente : historiquement, c'est l'État seul qui gère la politique étrangère, de sorte que la société civile ne saurait être crédible sur ce sujet. L'État possède en son sein les capacités d'analyse nécessaires et n'éprouve pas le besoin de consulter une expertise externe. Un haut fonctionnaire français aura spontanément l'impression de perdre son temps à aller écouter un universitaire ou à assister à un débat avec des représentants d'associations ou des journalistes. Ainsi en France ce sont les cabinets ministériels qui jouent un rôle similaire à celui des think tanks américains. Dans ces conditions, la situation en France est très difficilement comparable à celle des États-Unis. Les centres de recherche indépendants se sont créés relativement tardivement et luttent pour leur reconnaissance et leur crédibilité. Les conséquences de cet environnement peu propice sont clairement illustrées par le cas de l'Ifri (Institut Français de Relations Internationales). Si les apparences de l'Ifri semblent permettre une comparaison avec les institutions américaines, notamment le CFR, la réalité en est fort éloignée.

L'ifri a un parcours très différent du CFR et toute comparaison met en lumière les profondes différences du contexte culturel et politique. Non seulement l'Ifri est apparu beaucoup plus tard, mais surtout le projet a été personnalisé dès le départ. En 1973, un jeune polytechnicien, rentré de Berkeley il y a peu, Thierry de Montbrial, est chargé par le ministre des Affaires Étrangères, Michel Jobert, de créer, au sein du ministère, un centre d'analyse des relations internationales. Après avoir accompli sa mission et créé le CAP (Centre d'Analyse et de Prévision) - équivalent $\mathrm{du}$ «policy planning staff » aux États-Unis - Thierry de Monbrial créa un institut indépendant avec le soutien du Premier ministre de 
l'époque, Raymond Barre, en 1979 : l'Ifri. Ce nouvel institut reprenait une structure existante, le Centre d'Études de Politique Étrangère, créé en 1935 par des universités françaises et le Carnegie Endowment for International Peace. Ramsès et Politique étrangère constituent ses deux principales activités éditoriales. Politique étrangère, fondée en 1936, est la plus ancienne revue française en la matière. Cette revue trimestrielle donne une vision transversale des enjeux de l'actualité internationale. Depuis 1981, le rapport annuel Ramsès se consacre aux grandes tendances mondiales (environ 10000 exemplaires par an). Aux côtés de ces deux références, l'Ifri propose des publications plus courtes ou plus spécialisées. Parmi elles: les Notes de l'IFRI et Travaux et recherches de l'IFRI, les collections électroniques de l'Ifri et les livres des chercheurs.

Leconseild'administration regroupe entre autres, Robert Badinter (ancien ministre, sénateur), François Bujon de l'Estang (ambassadeur de France, président de Citigroup France), Michel Camdessus (gouverneur honoraire de la Banque de France, ancien directeur général du FMI), Jean-Claude Casanova (membre de l'Académie des sciences morales et politiques, directeur de la revue Commentaire), Alain Lamassoure (ancien ministre, député au Parlement européen), Christine Ockrent (journaliste), Louis Schweitzer (président d'honneur du conseil d'administration de Renault), et Simone Veil (ancien ministre d'État, ancien président du Parlement européen). Le directeur général est Thierry de Montbrial, membre de l'Académie des sciences morales et politiques. Le viceprésident secrétaire est Xavier de Villepin, sénateur honoraire, tandis que le vice-président trésorier est Pierre Joxe, ancien ministre, membre du conseil Constitutionnel. Ce conseil d'administration est doublé par un conseil stratégique, qui exerce une double fonction : stratégique, en réfléchissant aux orientations générales de la recherche; et scientifique, en portant un jugement sur la qualité des études et travaux de recherche. Certains de ses membres sont déjà dans le conseil d'administration, mais ce n'est pas le cas des suivants: Jean Klein (professeur émérite Paris I), Pierre Lévy (directeur du Centre d'analyse et de prévision au Ministère des Affaires étrangères), Dominique Moïsi (conseiller spécial - Ifri), Olivier Roy (directeur de recherche, CNRS) et Simon Serfaty (professeur à l'Université de Norfolk, titulaire de la chaire Brzezinski au CSIS de Washington).

L'Ifri est une association de loi 1901, dont le financement provient certes de sources diverses, mais l'État conserve un rôle fondamental ; on trouve ainsi, parmi ses soutiens : des subventions publiques (notamment de Matignon pour un peu plus de $40 \%$ de son budget) ou des contrats passés avec l'État, et ensuite des financements privés, les adhésions de personnes morales et physiques (il y aurait environ 500 membres), et des missions auprès de grandes entreprises. La liste de ses entreprises partenaires comprend : Axa, Air France, Arcelor, Sagem, Thalès, Suez, la Fondation Ford, BCG, LVMH, L'Oréal, l'Institut français du pétrole, au total une centaine d'entreprises seraient partenaires... Comme tous les instituts indépendants, l'Ifri attendait beaucoup de la loi Aillagon de 2003 visant à favoriser le mécénat, mais les conséquences tardent à se manifester ${ }^{6}$. Enfin, il convient de noter que l'Ifri est une 
petite structure, surtout en termes de recherche. L'équipe comprend en effet 80 personnes mais seulement 30 chercheurs. De ce point de vue, les dépenses administratives centrées autour de la direction prennent très largement le pas sur les activités de recherche.

Projet largement personnel, l'Ifri dépend donc aussi en grande partie du soutien de l'État. Qu'en est-il de ses missions? Elles reprennent les discours attendus : décloisonner l'approche des relations internationales et les mettre au cœur du débat public français. Pour mener cette ambition, ses travaux sont organisés en programmes couvrant différents domaines (espace, immigration, énergie, défense) et des pôles de recherche, dont trois sont constitués en centres ad hoc. D'abord indépendants de la direction centrale, ces trois centres ont été repris en main et ne possèdent maintenant qu'une indépendance toute nominale au sein de l'Ifri. Il s'agit de :

- Le Centre français sur les États-Unis (CFE) a été créé en 1999, en même temps que le Center of the United States and France (CUSF) à la Brookings institution à Washington DC, pour contribuer à une connaissance réciproque du fonctionnement des systèmes politiques, économiques, culturels et sociaux de part et d'autre de l'Atlantique. Il était dirigé jusqu'en 2006 par Guillaume Parmentier, Professeur associé à Paris 2. Mais depuis 2006, le CFE est une coquille vide au sein de l'Ifri qui ne possède donc plus d'expertise sur les États-Unis, ni de lien privilégié avec la Brookings, dans la mesure où le CUSF est devenu le Center of the United States and Europe en 2004.

- Le Centre Asie, créé en 2001, consacre l'essen- tiel de ses travaux à l'Asie Orientale en liaison étroite avec ses homologues asiatiques, européens et américains. Le Centre travaille à une expertise comparative du Nord-Est et du Sud-Est asiatique ainsi que des enjeux multilatéraux et bilatéraux de la zone. Après avoir été créé et dirigé par François Godement, il est dirigé depuis 2005 par Valérie Niquet.

- Le Comité d'études des relations franco-allemandes (Cerfa) est un cas à part dans la mesure où sa création est liée à un accord franco-allemand de 1954. Il est sous la tutelle conjointe de l'Ifri et de son homologue allemand, la DGAP (Deutsche Gesellschaft für Auswärtige Politik). Le secrétariat général est assuré depuis 1991 par Hans Stark.

Depuis juin 2005, l'Ifri a créé une antenne à Bruxelles: IFRI Bruxelles. Il entend élargir son champd'influenceetsondomainedecompétence. L'Institut organise les "Mardis de l'IFRI à Bruxelles », rendez-vous hebdomadaire sur les thèmes liés à l'agenda européen. L'Ifri cherche aussi à travailler régulièrement en partenariat avec ses homologues à l'international, mais ses relations demeurent très largement ponctuelles.

Jean Monnet avait déclaré que "Rien n'est possible sans les hommes mais rien n'est durable sans les institutions $\gg$. Les deux exemples du CFR aux États-Unis et de l'Ifri en France confirment pleinement cette citation. Mais si le CFR a réussi à s'implanter durablement, c'est grâce à une montée en puissance collective. Elihu Root, ou les autres personnalités derrière le lancement du CFR, se sont effacées le moment venu. Dans le cas de l'Ifri, et derrière une rhétorique similaire 
à celle que l'on peut trouver outre-Atlantique, le fonctionnement demeure bien trop personnalisé, ce qui est peut-être inévitable dans un contexte français peu propice aux centres de recherche indépendants, mais qui, liant trop étroitement l'Ifri à son fondateur, n'est pas de bon augure pour la pérennité de l'Ifri.
$\mathrm{N} \cdot \mathrm{O} \cdot \mathrm{T} \cdot \mathrm{E} \cdot \mathrm{S}$
1. Alexis de Tocqueville, De la démocratie en Amérique, tome 2, deuxième partie, chap. 5, p. 137, Paris, Gallimard, 1982 (1835). La citation continue : «Les Américains de tous les âges, de toutes les conditions, de tous les esprits, s'unissent sans cesse.... Partout où, à la tête d'une entreprise nouvelle, vous voyez en France le gouvernement et en Angleterre un grand seigneur, comptez que vous apercevrez aux États-Unis une association ».

2. Justin Vaisse, « Petit guide des revues et thinktanks : les très riches heures des relations internationales aux États-Unis ", dans Revue Internationale et Stratégique, n 34, été 1999.

3. Elihu Root (1845-1937) est une figure majeure de la politique américaine du début $\mathrm{du} \mathrm{XX}^{\mathrm{e}}$ siècle, représentant le « progressisme » républicain de cette période. Il fut secrétaire d'État de 1899 à 1909 puis sénateur républicain de l'État de New York (19091915).

4. Par exemple le livre de Laurence H. Shoup et William Minter, Imperial Brain Trust : the Council on Foreign Relations and United States Foreign Policy, New York, Monthly Review Press, 1977.

5. Sur la spécificité de ce statut et ses implications, je renvoie à l'ouvrage de Frédéric Martel, De la culture en Amérique, Paris, Gallimard, 2006, notamment les pages 331-339.

6. La loi relative au mécénat, aux associations et aux fondations a été votée le 1er août 2003. Cette loi modifie notamment la loi de 1901 sur les associations et le Code général des impôts (CGI) pour développer le mécénat, réformer la reconnaissance d'utilité publique et harmoniser les dispositifs applicables aux dons. Les réductions d'impôts pour les particuliers (revenu) et les entreprises (impôt sur les sociétés) sont 
relevées dans le cadre des dons faits à des associations reconnues d'utilité publique. La loi visait à doter la France d'un système comparable à celui d'autres pays européens, notamment la GB (les charity trusts) et l'Allemagne (Stiftung). On reste loin, en tous les cas, des possibilités offertes aux États-Unis.
$R \cdot E \cdot S \cdot U \cdot M \cdot E ́$

Cette brève monographie compare le développement d'un des plus anciens think tanks américain, le Council on Foreign Relations et l'Institut Français des Relations Internationales (Ifri). Le texte illustre ainsi les profondes divergences entre les deux institutions, notamment au niveau de leur direction qui, dans le cas de l'Ifri, reste très personnalisée.

This brief overview of two leading institutions in the field of policy-oriented research - the Council on Foreign Relations (CFR) in the US and the Institut Français des Relations Internationales (Ifri) - emphasizes the deep differences between their respective developments. Unlike CFR, which successfully managed to evolve into a collective research entity, Ifri is still very much related to its founder, thus running the risk of being short-lived. 